\begin{tabular}{|c|c|}
\hline 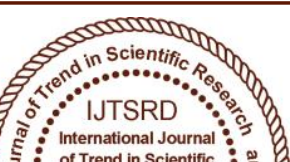 & $\begin{array}{l}\text { International Journal of Trend in Scientific } \\
\text { Research and Development (IJTSRD) }\end{array}$ \\
\hline 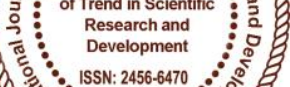 & International Open Access Journal \\
\hline intivis & ISSN No: 2456 - 6470 | www.ijtsrd.com | Volume - 2 | Issue -3 \\
\hline
\end{tabular}

\title{
Monitoring of the Chemical Oxygen Demand and Identify the Presence of Gas
}

\author{
${ }^{1}$ J Giriprasad, ${ }^{1}$ K Kumaraguru , ${ }^{1} \mathrm{~L}$ Mohammad Shifath Ali, ${ }^{2}$ Mrs Audline Beena, \\ ${ }^{3}$ Dr. D Rajinigirinath \\ ${ }^{1}$ Student, ${ }^{2}$ Assistant professor, ${ }^{3}$ Head of the department, \\ Department of Computer Science and Engineering, \\ Sri Muthukumaran Institute of Technology, Chennai, Tamil Nadu
}

\begin{abstract}
Water pollution is one of the biggest fears for the green globalization. In order to ensure the safe supply of the drinking water the quality needs to be monitor in real time. In this paper we present a design and development of a low cost system for real time monitoring of the water quality. The system consist of several sensors is used to measuring physical and chemical parameters of the water. The parameters such as temperature, DO, Turbidity, conductivity and $\mathrm{PH}$ of the water can be measured. The measured values from the sensors can be processed by the core controller. Finally, the sensor data can be processed and send to the system.
\end{abstract}

Keywords: Water quality monitoring, S COD parameter, Soft measurement, Neural Network

\section{INTRODUCTION:}

For most control process, because the on-line analysis appearance not only price is expansively, the maintenance is maintained complicatedly, especially stagnant not greatly afterwards, leads to controlling quality to descend, and is hard to the satisfied production requirement. The soft measurement technology [2] [9] [10] apply the computer technology to measure some important variables which are hard to or cannot real-time measure using some easy measuring variables, and by way of forming certain mathematics concerns. This kind of method [3] use software to substitute for the sensor function. Its response is rapid, and the leading variable In the light of this important issue, the softsensing method based on neural network for wastewater treatment is proposed with the Application of model artificial intelligence information can continuously gives. Their invests is lower and the maintenance is simple. The process control of wastewater treatment has been the focus of study in recent years. The technology of ambiguity control has been successfully applied in feed-water treatment in Japan, anaerobic digestion treatment in U.S.A. and etc. But, because the wastewater quality parameters can't be detected on real-line presently, open loop control or other control based on indirect parameters are taken in many methods, which affects the control effect. Thus, how to get the wastewater quality parameters online has been the key to improve the control effectiveness. Technology for the purpose of resolving the real-time detecting problem of wastewater treatment process. Many methods have been put forward concerning wastewater monitoring. Sewer gas is a complex mixture of toxic and nontoxic gases produced and collected in sewage systems by the decomposition of organic household or industrial wastes, typical components of sewage. Sewer gases may include hydrogen sulfide, ammonia, methane, esters, carbon monoxide, sulfur dioxide and nitrogen oxides.

It is important to recognise that gas detectors are not simply installed for process monitoring - they are required to save lives. However, this life saving equipment must also be robustly constructed to survive the rigours of deployment in a water plant. 
The rapid development of Information Technology in recent years has injected new vitality into distance learning. At present the desktop computer is still the most popular hardware being used all over the world where distance learning is concerned. with the popularity of portable devices using the Android operating system and the technology of the Internet of things, the problems will eventually be solved completely.

\section{ANDROID SYSTEM}

Android is a Linux-based operating system [1] designed primarily for touch-screen mobile devices such as smart phones and tablet computers. Initially developed by Android, Inc., which Google backed financially and later bought in 2005, Android is open source and Google releases the code under the Apache License.[2]. Permissive licensing allows the software to be freely modified and distributed by device manufacturers, wireless carriers and enthusiast developers. Android operating system has rich resources of hardware and software applications.

\section{EXISTING SYSTEM}

Although planning is a crucial part of the system development process, it is often neglected by project managers. The problem being addressed by this paper is that of inadequate models for planning the requirements capture and analysis stage (RCA) of a software development project. It is stressed that there is a need for a new model because the existing models give inaccurate, inconsistent or unreliable predictions. Additionally, they are based on either inappropriate variables or variables that cannot be measured at the beginning of the development process. Finally, existing models do not support the planning of individual stages of the development process but only try to make predictions about the project development process as a whole. This paper examines existing models and provides evidence about their inadequacy and lack of accuracy, and then introduces a new model and presents the approach followed for its development. China has obtained certain growth in the domain of water pollution monitoring technology but mainly relying on foreign experience. Moreover, water quality online monitoring system has not been widely applied. In addition, the online measurement of some water quality parameters is too expensive to be popularized absolutely. the model did not achieve satisfactory results for the measurement of NO3-N due to erroneous selection of auxiliary variables.

\section{PROPOSED SYSTEM}

China has obtained certain growth in the domain of water pollution monitoring technology but mainly relying on foreign experience. Moreover, water quality online monitoring system has not been widely applied. In addition, the online measurement of some water quality parameters is too expensive to be popularized absolutely. the model did not achieve satisfactory results for the measurement of NO3-N due to erroneous selection of auxiliary variables.

The water quality parameters $\mathrm{pH}$ measures the concentration hydrogen ions. It shows the water is acidic or alkaline. Pure water has $7 \mathrm{pH}$ value, less than $7 \mathrm{pH}$ has acidic, more than $7 \mathrm{pH}$ has alkaline. The range of $\mathrm{pH}$ is $0-14 \mathrm{pH}$. For drinking purpose it should be $6.5-8.5 \mathrm{pH}$. Temperature sensor measures how the water is, hot or cold. In this proposed block diagram consist of several sensors (temperature, $\mathrm{pH}$ ) is connected to core controller. The core controller are accessing the sensor values and processing them to whether water could be purify or not.

\section{SYSTEM ARCHITECTURE}

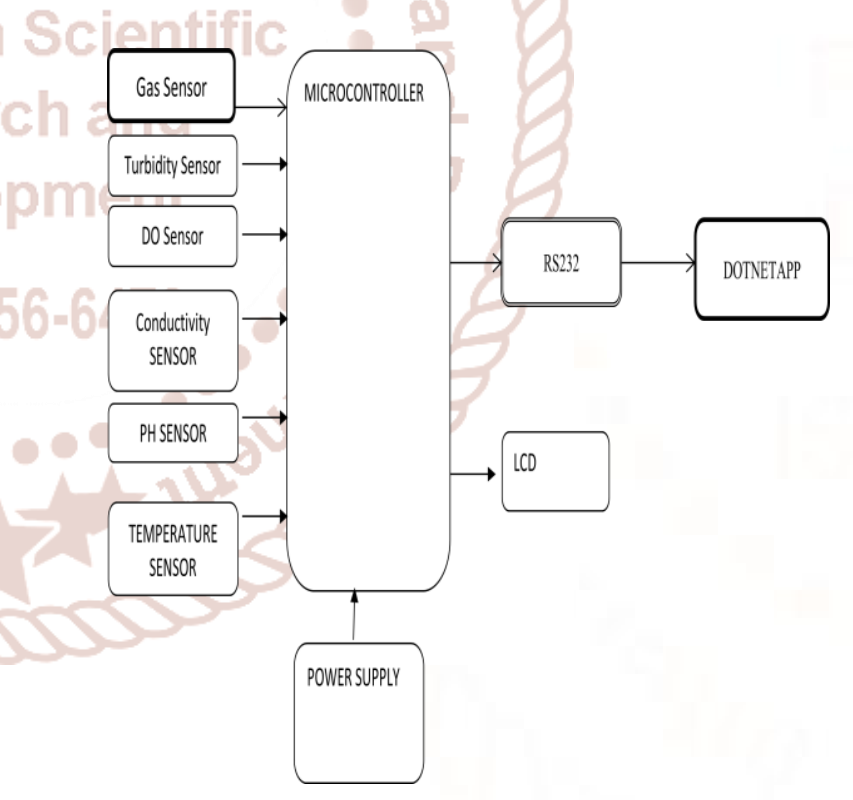

The core circuit board acts as the main control unit of the entire device and performs the functions of operation. The main function of the backplane is data acquisition and system power supply. Android system is operating environment of the application software. During operation, the system $\square$ realizes the fast and real-time measurement of conventional five water quality parameters through the sensor mounted on the backplane. After that, it uses soft measurement techniques to measure COD (Chemical Oxygen 
Demand) based on the measured value of $\mathrm{pH}$, dissolved oxygen, temperature, turbidity, gaseous and electrical conductivity. At the same time, all the data will be stored.

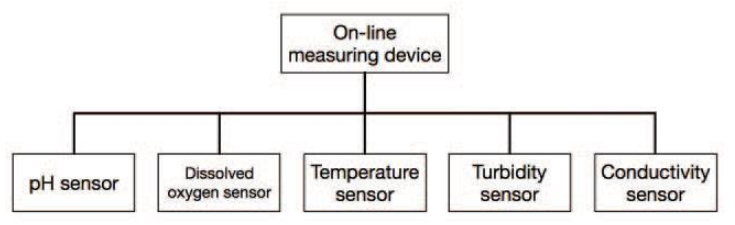

\section{NEURAL NETWORK}

\section{A. ANN Architecture}

An ANN is a highly interconnected network of man simple processing units called neurons, which are analogous, the biological neurons in the human brain. An ANN normally consists of three layers, an input layer, a hidden layer and an output layer. An important step in developing an ANN model is the determination of its weight matrix through training. There are primarily two types of training mechanisms, supervised and unsupervised. A supervised training algorithm requires an external teacher to guide the training process. The primary goal in supervised training is to minimize the error at the output layer by searching for a set of connection strengths that cause the ANN to produce outputs that are equal to or closer to the targets. A supervised training mechanism called back-propagation training algorithm (BP) is normally adopted in most of the engineering applications.
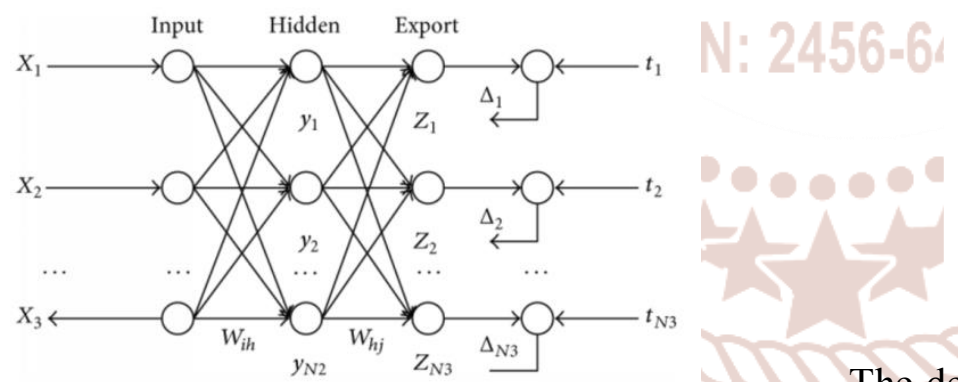

the next sampling instant, but the change of that signal, that is $\boldsymbol{\Delta r}^{\wedge}(\boldsymbol{k}+\mathbf{1})$. And after that, the farther predicted value of this signal is given by calculating the following equation as

$$
r^{\wedge}(k+1)=r(k)+r^{\wedge}(k+1)
$$

\section{B. BP NETWORK}

The BP network [3][7] is composed by one input layer and one or more hidden layers and one output layer. The learning course of network includes two courses, one is the input information transmitting forward directed and another is the error transmitting backward directed. In the transmitting forward direction, the input information goes to the hidden layers from input layer and goes to the output layer. If the output of output layer is different with the wishful output result then the output error will be calculated, the error will be transmitted backward directed then the weights between the neurons of every layers will be modified in order to make the error become minimum. The key of $\mathrm{BP}$ network is the error transmitting backward directed of learning course. The course is accomplished through minimize an object function that is the error sum of squares between the actual output of network and the expectant output. BP network is shown in below:

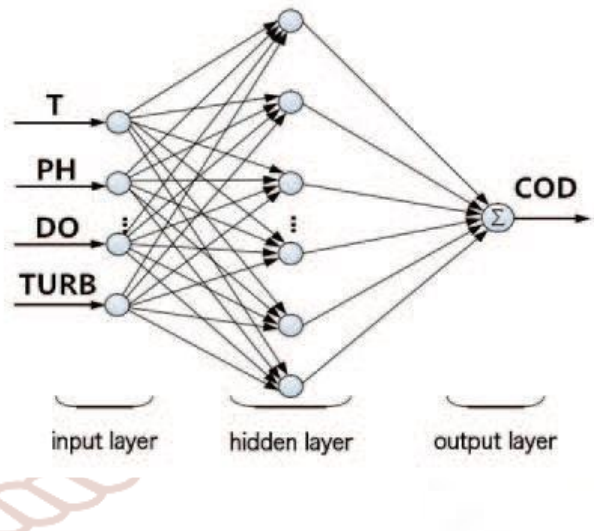

The design work of the algorithm is divided into four parts. First of all, Use the dynamic ASM model based A three-layer NN shown in Figure is used to construct the signal prediction system, and the aim is to use historical sample data to predict the next sampled signal. The model architecture, training method, and training rates were determined using a trial-and-error approach. The inputs of the NN are $\boldsymbol{r}(\boldsymbol{k}) 、 \boldsymbol{r}(\boldsymbol{k})-\boldsymbol{r}(\boldsymbol{k}$ -1) 、 $\boldsymbol{r}(\boldsymbol{k}-\mathbf{1})$ and $\boldsymbol{r}(\boldsymbol{k}-\mathbf{1})-\boldsymbol{r}(\boldsymbol{k}-2)$. The hidden layer contains 5 neurons. In addition, S-type active function is chosen as hidden layer and linear active function is chosen as output layer. To reduce the impact on training result caused by learning speed, the output of the $\mathrm{NN}$ is not the predicted signal $\boldsymbol{r}^{\wedge}(\boldsymbol{k}+1)$ at on activated sludge method and cell death, regeneration and maintenance theory to establish neural network model. Secondly, obtain experimental data and process these data through the experiment on COD Degradation of Industrial Wastewater. And then use the experimental data for 960 network training and network verification. Finally, transplant the algorithm to make it conform to JNI specification, so that it can work with Java code. 


\section{DESIGN OF NEURAL NETWORK:}

The ASM1 [4] model mainly contains 13 sub-reaction processes, 19 parameters, 5 stoichiometric numbers and 14 kinetic parameters.

Combined with the model based on mechanism of activated sludge process and the factor affecting filamentous bacteria grow in aeration tank is taken into account in the selection and analysis process of auxiliary variables.

$$
C_{T O D}=S_{I}+S_{S}+X_{I}+X_{S}+X_{B, H}+X_{B, A}+X_{P}
$$

$C_{T O D}$ represents the total organic content of the sewage; $S_{I}$ represents soluble inert organic matter; $S_{S}$ represents biodegradable organic matter; $X_{I}$ represents granular inert organic matter; $X_{S}$ represents slow biodegradable organic matter; $X_{B, H}$ represents the concentration of anaerobic bacteria; $X_{B, A}$ represents the concentration of autotrophic bacteria; $X_{P}$ represents biological attenuated granular products. The importance of the individual components is not exactly equal. According to the actual measurement, it is found that $X_{B, A}$ and $X_{P}$ are negligible because of their small content. In addition, $X_{B, H}$ can also be ignored or combined with $X_{S}$ to calculate.

By analyzing the growth of filamentous bacteria, we summarize the formula as follows:

For the general organic matter, bio-oxidation for breathing about $1 / 3$ of the energy generated the

synthesis of cell material accounts for about $2 / 3$ of the energy. After the endogenous breath, the residue is about $20 \%$ of the cell material.

To get the relationship as follows:

$(B O D)_{U} \approx(1 / 3) C O D_{B}+(2 / 3) * 0.8 C O D_{B}=.87 C O D_{B}$

$C O D_{B}$ represents the biodegradable component in the chemical oxygen demand, $(B O D)_{U}$ represents the total biochemical oxygen demand.

$(B O D)_{5} \approx(2 / 3) \quad(B O D)_{U} \quad(B O D)_{5} \approx$ $(2 / 3) * 0.87 C O D_{B} \approx .58 C O D_{B}$

Then get the following relationship: $(B O D)_{5} \approx$ $.58 C O D_{B}$

Therefore, $\mathrm{BOD}[8]$ and COD have a great relevance according to McKinney's [6] assumption. As COD are usually measured using chemical methods, electrochemical methods, photochemical methods and so on.

$$
u_{f}=f_{2}\left(u_{m, f}, F / M, M L S S, S_{O}, S_{N}, S_{P}\right)
$$

$u_{m, f}=f_{3}(T, P H)$

$u_{f}$ is the specific growth rate of filamentous bacteria, $u_{m, f}$ is the maximum growth rate of filamentous bacteria, $f$ is an unknown function, $F / M$ is the load of sludge. From the formula above we can conclude that the growth rate of filamentous bacteria is related to the variable quantities involved in the formula.

\section{EMBEDDED SOFTWARE DESIGNOF SOFT MEASUREMENT IN ANDROID OS:}

The neural network is a BP neural network $[1,4]$ with four inputs, one output. The four inputs correspond to the temperature, $\mathrm{PH}$, dissolved oxygen and turbidity parameters measured by the sensor, and an output corresponds to the predicted COD parameter $[1,3]$. The 480 sets of standard data for training are derived from the industrial wastewater COD degradation experiments mentioned above, of which 380 sets of data used to train, 100 sets of data used to test. First of all, use the standard data from the above-mentioned 380 sets of standard data to train the network. Then we will put 100 sets of standard data into the network for comparison. When the measured error meets the design requirements, this network will be usable. Next we save the trained network as a custom function of MATLAB and use the MATLAB Coder toolbox [6] to convert the saved function into a $\mathrm{C}$ program that does not depend on the specific controller. In this case, it is necessary to realize that the system is running on the Android and the device controller is the ARM architecture controller, thus the above method can only be realized theoretically.

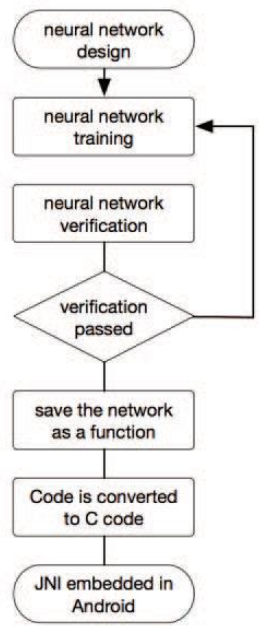

\section{MODULE:}

\section{A. POWER SUPPLY: -}

The power supply output is given to microcontroller and other circuit also; the design of the power supply is mainly because of the micro controller, the micro controller work in Dc source with a voltage of $+5 \mathrm{v}$. 
As we are getting the line voltage VL has $230 \mathrm{v}$ in ac source, so it is not possible. This power supply designs an output of $+5 \mathrm{v}$ Dc to activate the micro controller.

POWER SUPPLY +5V
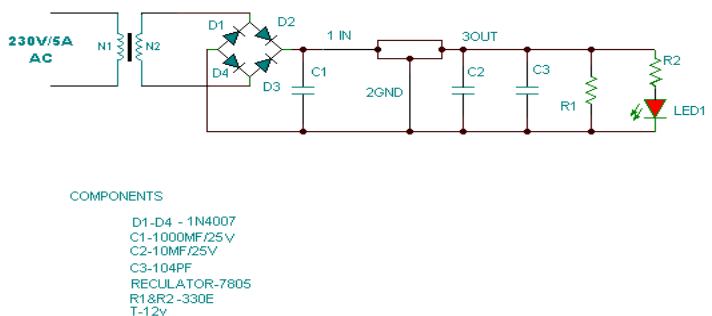

\section{B. Collect Sensor Information}

The micro controller, which we are using here, is PIC 16F877 .It consists of 5 ports, ADC, CLK\& MCLR. These are inbuilt with in 40 pins. The micro controller accepts and gives the $\mathrm{o} / \mathrm{p}$ in digital form. In this project we use five sensors such as temperature, turbidity, DO Sensor, Conductivity sensor and $\mathrm{PH}$ sensor. These sensors information are collected by the microcontroller.

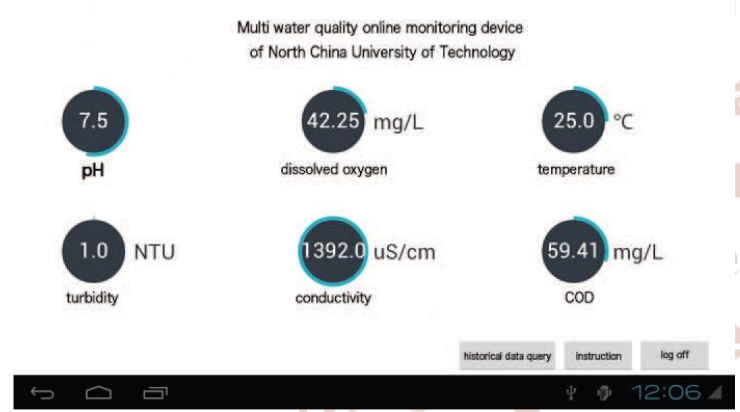

\section{COMPONENTS}

\section{- TEMPERATURE SENSOR:}

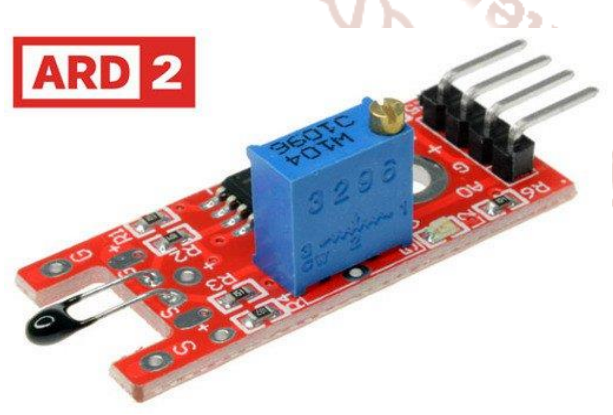

A thermistor is a ceramic semiconductor which exhibits a large change in resistance with a change in its body temperature. The word thermistor is actually a contraction of the words "THERMAL RESISTOR".

Although there are both positive coefficient (PTC) and negative coefficient (NTC) are available, for our application we use negative coefficient (NTC) type thermistor. These NTC thermistors are compose of oxides such as the oxides of the MANGANESE, NICKEL, COBALT, COPPER, IRON and TITANIUM.

The thermistors have much better sensitivity than RTD's and are therefore better suited for precision temperature measurements. The availability of high resistance values allows the thermistors to be used with long extension leads, since the lead resistance or contact resistance effects can be greatly diminished.

\section{B. Turbidity Sensor}

Turbidity sensor module

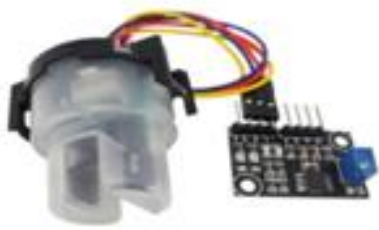

Turbidity is the quantitative measure of suspended particles in a fluid. Turbidity is a measure of water's lack of clarity and is an important indicator of water quality. Water with high turbidity is cloudy, while water with low turbidity is clear. The cloudiness is produced by light reflecting off particles in the water; therefore, the more particles in the water, the higher the turbidity. High turbidity can be detrimental to water quality as more sunlight is absorbed, causing an increase in water temperature.

\section{DISSOLVED OXYGEN SENSOR}

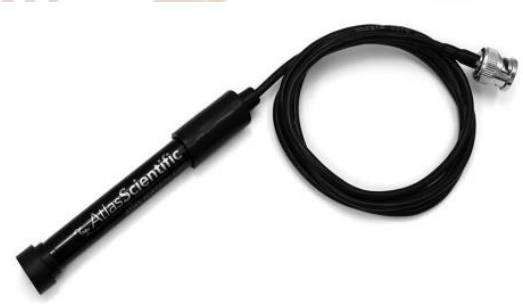

For aquatic species, adequate dissolved oxygen is of prime importance to their continued survival. Since dissolved oxygen levels are directly related to good water quality, the two are highly interdependent. Many factors can affect DO levels, and an understanding of these levels in order to make informed decisions concerning wastewater treatment operations, hypoxic zones, aquaculture facilities or large-scale ecosystems is essential. 


\section{CONDUCTIVITY SENSOR}

Conductivity is the measure of a solution's ability to pass or carry an electric current.

The term Conductivity is derived from Ohm's Law,

$$
E=I 2 * R
$$

Where Voltage (E) is the product of Current (I) and Resistance (R); Resistance is determined by Voltage/Current.

When a voltage is connected across a conductor, a current will flow, which is dependent on the resistance of the conductor.

Conductivity is simply defined as the reciprocal of the Resistance of a solution between two electrodes. The measurement of specific conductivity in aqueous solutions is becoming increasingly important for the determination of impurities in water or the concentration measurement of dissolved chemicals.A conductivity probe consists of an electrode pair, to which a voltage is applied. The conductivity sensor measures the flowing current and calculates the conductivity.

\section{E. PH SENSOR}

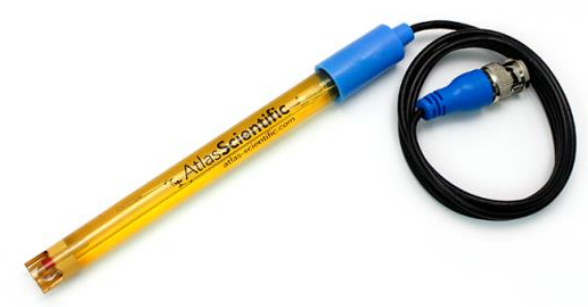

$\mathrm{pH}$ is the numeric representation of gram-equivalent per liter of hydrogen ion concentration in any solution. It varies between 0 to 14. It is the logarithmic measurement of moles of hydrogen ions per liter of solution. The solutions having $\mathrm{pH}$ value between 0 to 7 are acidic solutions with large concentration of hydrogen ions whereas solutions having $\mathrm{pH}$ value between 8 to 14 are basic solutions with small hydrogen concentration. The solutions having $\mathrm{pH}$ value of 7 are neutral solutions. Measuring the $\mathrm{pH}$ gives the measure of alkalinity or acidity of a solution.

\section{F. GAS SENSOR}

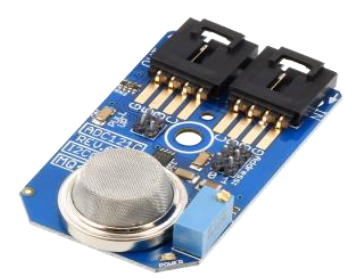

Gas sensors are available in wide specifications depending on the sensitivity levels, type of gas to be sensed, physical dimensions and numerous other factors. When a gas interacts with this sensor, it is first ionized into its constituents and is then adsorbed by the sensing element. The gas sensor module consists of a steel exoskeleton under which a sensing element is housed. This sensing element is subjected to current through connecting leads. This current is known as heating current through it, the gases coming close to the sensing element get ionized and are absorbed by the sensing element.

\section{CONCLUSION}

The improved simulated annealing neural network is applied to soft measurement modeling of sewage treatment, it combines the advantages of both SA and $\mathrm{BP}$ network. A set of multi-water quality real-time online measurement device has been designed based on embedded system and neural network algorithm. The work of hardware design and software design of the device has been completed successively. The works of hardware design include the design and welding of the core board and the backplane.

The soft measurement modeling method can truly detect and assess the quality of sewage treatment in real time by learning the sewage treatment parameter information of sensors acquired, thus the real time control for the quality of sewage treatment can be implemented. The experimental results show that this method is feasible and effective and this soft measurement modeling method has good application foreground in the sewage treatment.

The $\mathrm{pH}$ of the sample reduced to 7.3 from 7.4 slightly reduced in the $\mathrm{pH}$ value. The COD of the sample is decrease to 768 from 896 in 48 hour of inverse fluidization. The BOD of the sample is decrease to 276 from 324 in 48 hour of inverse fluidization. The observed reduction in COD, BOD and $\mathrm{pH}$ indicate the suitability for the application of inverse fluidised bed for waste water treatment for any industry.SCOPE OF FUTURE WORK Treatment of industrial effluents can be done

(i) By using different bed heights of same sized polypropylene beads. (ii) By using different sizes of the beads. (iii) by using different concentrations of effluent (iv) by varying air flow rates (v) by continuous flow of effluents. 


\section{REFERENCE}

1. PENG Yong-zhen. "Five Advantages of SBR Process".

2. Wang Qun. Research on on line monitoring system of sewage based on ARM and GPRS School of electrical engineering and information engineering, Lanzhou University of Technology, 2011.

3. Qing Xiaoxia, Yu Jianping, "Soft sensors and it's use in wastewater treatment systems," Industrial Water Treatment, vol. 25, no. 3, pp. 13-16, March 2005.

4. Guo Nan. Research on BOD soft sensor based on Neural Network [D]. Beijing University of Technology,2014.

5. Deng Rongsen. oxidation channel waste water treatment principle and technology. Beijng: Chemical Industry Press, 2006.

6. Ren DongHong. Soft sensor model for effluent quality of sewage treatment based on integrated neural network[D]. Beijing University of Technology,2013.

7. Luo TengFei. Prediction of effluent from wastewater treatment based on improved BP neural network [D]. School of municipal engineering, Inner Mongolia Agricultural University, 2012.

8. Guo Nan. Design of BOD soft sensor based on Neural Network[D]. School of electronic information and control engineering, Beijing University of Technology, 2014.

9. Qing Xiaoxia, Yu Jianping, "Soft sensors and it's use in wastewater treatment systems," Industrial Water Treatment, vol. 25, no. 3, pp. 13-16, March 2005.

10. WANG Zhengxiang, LIU Zaiwen, XUE Fu xia, "Soft Sensing Technique for Sewage Treatment Process," Journal of Beijing Techno logy and Business University (Natural Science Edition), vol. 23, no. 3, pp. 31-34, 2005.

11. Harold H. Szu and Ralph L. Hartley, Nonconvex Optimization by Fast Simulated Annealing, Proceedings of the IEEE, vol. 75, no. 11,pp.15381540, 1987.

12. Tian Jingwen, Gao Meijuan, Thin Interbedded Reservoir Parameters Predicting Based On Improved Simulated Annealing Artificial Neural
Network, Information and Control, vol.31, no.2, pp.180-188, 2002.

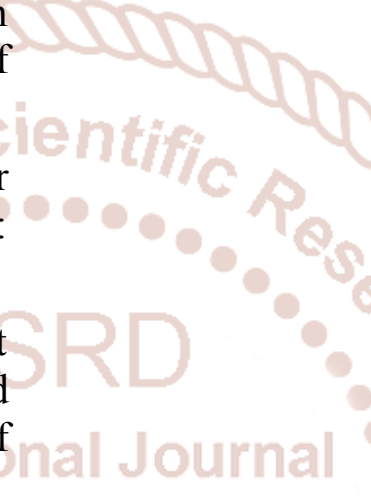

